\title{
ATLAS results and prospects with focus on beyond the Standard Model ${ }^{\text {th }}$
}

\author{
Lucia Masetti, on behalf of the ATLAS Collaboration
}

PRISMA Cluster of Excellence and Institute for Physics, Johannes Gutenberg University, Mainz, Germany

\begin{abstract}
A summary of results relevant for searches of phenomena beyond the Standard Model (SM) obtained by the ATLAS experiment at the LHC is presented. While all SM measurements can be considered as indirect searches, the focus will be set on direct searches and measurements interpreted as limits on phenomena beyond the SM. Prospects towards Run 3 and High Luminosity LHC are reported where available. Most results are based on an integrated luminosity of $36 \mathrm{fb}^{-1}$ at a centre-of-mass energy of $13 \mathrm{TeV}$.
\end{abstract}

\section{Introduction}

The Standard Model (SM) of particle physics is an extremely successful description of the phenomena observed so far. It leaves however several open questions, e.g. concerning the nature of Dark Matter (DM), the matter-antimatter asymmetry and the hyerarchy problem. Many theories and models beyond the SM (BSM) provide answers to these questions and are therefore tested experimentally. At the LHC the high collision energy is used to search directly for heavy new particles, while the high luminosity is exploited in searches for rare processes. Results are generally presented in a model independent way leaving the possibility to recast them for new models.

During the current data taking period (Run 2) more than $100 \mathrm{fb}^{-1}$ of data have already been collected. In Run 3 (2021-2023) the centre-of-mass energy will be increased to $14 \mathrm{TeV}$ and an integrated luminosity of 300 $\mathrm{fb}^{-1}$ is expected, while the High Luminosity LHC (HLLHC) will increase the total integrated luminosity by one order of magnitude at the same energy. To face the challenges of the upcoming Run 3 and at the HL-LHC, most of all in terms of trigger rate and multiple collision (pile-up) rejection, the ATLAS detector [2] needs

\footnotetext{
${ }^{2}$ Copyright 2018 CERN for the benefit of the ATLAS Collaboration. Reproduction of this article or parts of it is allowed as specified in the CC-BY-4.0 license
}

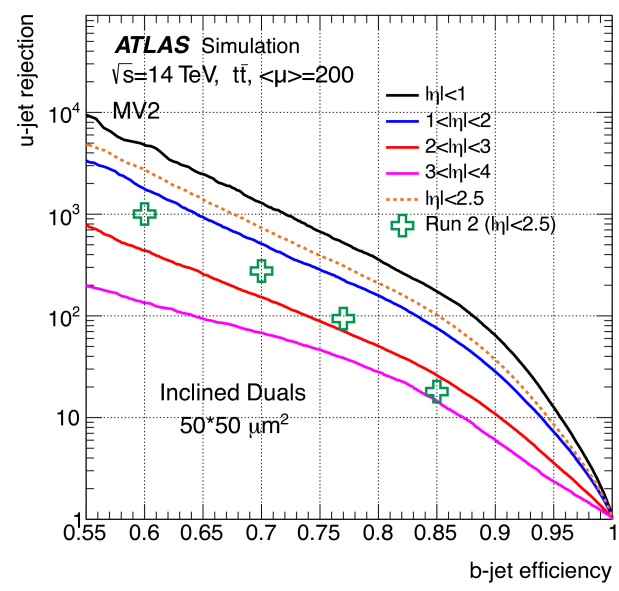

Figure 1: Performance of the MV2 b-tagging algorithms in $\bar{t} t$ events with 200 pile-up for the ITk layout. The rejection of light jets for different $\eta$ regions is shown as a function of b-jet efficiency. For comparison purposes, the performance for ATLAS during Run 2 with an average of 30 pile-up events is shown as crosses [1]. 
to be upgraded with new muon detectors, a new inner tracker (ITk), as well as improved read-out and trigger electronics. The new hardware together with new reconstruction techniques allow to recover or improve on the Run 2 performance in spite of a much higher pileup level. As an example, the expected $b$-tagging performance at the HL-LHC is shown in Figure 1. In the same range of pseudorapidity $(\eta)$ the light-quark rejection at a given efficiency is significantly higher than in Run 2 . The larger coverage of the ITk allows for $b$-tagging up to $|\eta|<4$.

\section{Direct BSM searches}

Direct searches for BSM particles are presented in this Section, divided into dedicated searches for supersymmetric (SUSY) particles in 2.1 and signature-based and DM searches in 2.2.

\subsection{SUSY searches}

Several SUSY searches are dedicated to the pair production of top squarks $(\tilde{t})$. Since the decay topology de-

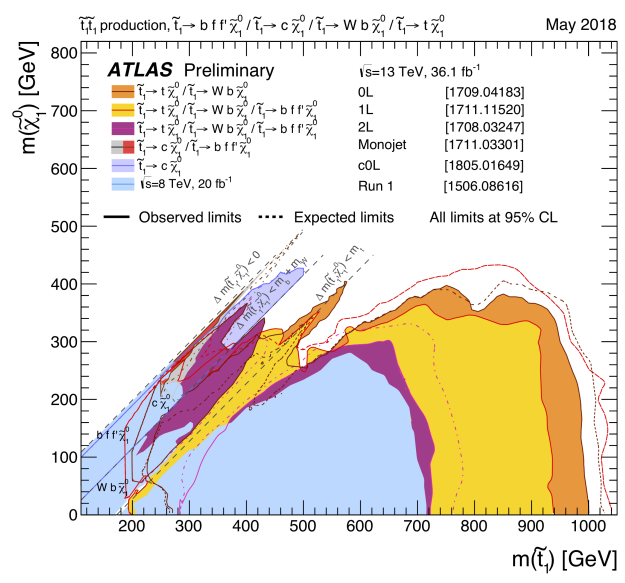

Figure 2: Summary of the dedicated ATLAS searches for top squark (stop) pair production based on $36 \mathrm{fb}^{-1}$ of $p p$ collision data taken at $\sqrt{s}=13 \mathrm{TeV}$. Exclusion limits at $95 \% \mathrm{CL}$ are shown in the $\widetilde{t}_{1}-\widetilde{\chi}_{1}^{0}$ mass plane. The dashed and solid lines show the expected and observed limits, respectively, including all uncertainties except the theoretical signal cross section uncertainty (PDF and scale) [3].

pends on the masses of the intermediate SUSY particles, dedicated signal regions are chosen for each scenario, while the background estimate is performed in appropriate control regions. So far no evidence for signal is observed and model-independent limits on the number of signal events are reported, as well as their interpretation in terms of limits on the SUSY particle masses. Currently the precision of the results is limited by the available statistics in the signal regions and by background modelling uncertainties of the order of $20 \%$. A recently published result in the almost degenerate region $\left(\Delta m=m_{\widetilde{t}_{1}}-m_{\widetilde{\chi}_{1}^{0}}<m_{W}+m_{b}\right)$ addresses the $\widetilde{t}_{1} \rightarrow c \widetilde{\chi}_{1}^{0}$ decay by using a dedicated $c$-tagging algorithm [4]. The result is included in the summary shown in Figure 2. Additional searches aim at $\widetilde{t}_{1}$ decays to a $\widetilde{\tau}_{1}$ [5] or to jets in an R-parity violating (RPV) model [6] and exclude them at $95 \%$ confidence level up to $m_{t_{1}}=1.16 \mathrm{TeV}$ and $m_{t_{1}}=410 \mathrm{GeV}$, respectively. At the HL-LHC a discovery of the top squark in the decay $\widetilde{t}_{1} \rightarrow \widetilde{\chi}_{1}^{0}$ will be possible up to $m_{t_{1}} \simeq 500 \mathrm{GeV}$ [7].

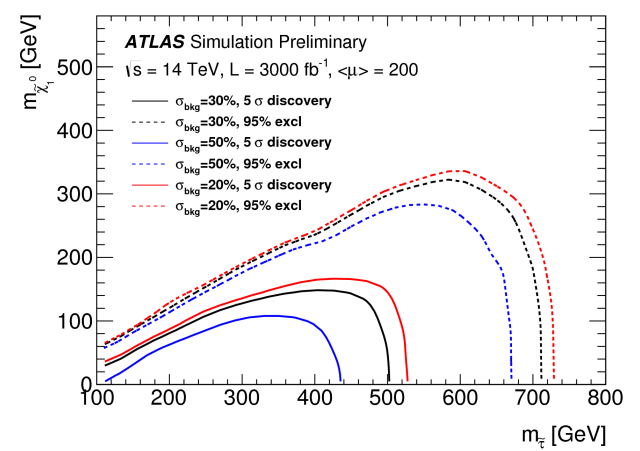

Figure 3: The 95\% CL exclusion limits and $5 \sigma$ discovery contours for $3000 \mathrm{fb}^{-1}$ luminosity on the combined $\widetilde{\tau}_{L} \widetilde{\tau}_{L}$ and $\widetilde{\tau}_{R} \widetilde{\tau}_{R}$ production at the HL-LHC with different systematic uncertainty assumptions on SM backgrounds [8].

Considering the superpartners of the electroweak bosons and the production of sleptons, very different processes can lead to similar final states. Using a similar general strategy as for the squark searches, the focus of these analyses lies mostly on the background estimate techniques. Both R-parity conserving (RPC) and RPV scenarios are considered. A recent search with 2 and 3 lepton signatures uses a $\gamma+$ jets template to estimate the dominating $Z+$ jets background [9], while a 4 lepton analysis uses a data-driven method to estimate the background from fake or non-prompt leptons [10]. No significant excess above the SM expectation is observed in any of the signal regions considered, and the results are used to calculate exclusion limits at $95 \%$ confidence level in several simplified model scenarios. The sensitivity for discovery and exclusion at the HL-LHC has been studied in [11] and [8]. The results of the latter publication in the $\left(m_{\tau}, m_{\widetilde{\chi}_{1}^{0}}\right)$ plane are shown in Figure 3.

In case of mass-degenerate spectra, small couplings or highly virtual intermediate states, the new particles can be long-lived and their search requires dedicated reconstruction techniques. Additionally to the limited statistics, the background estimate is therefore particu- 


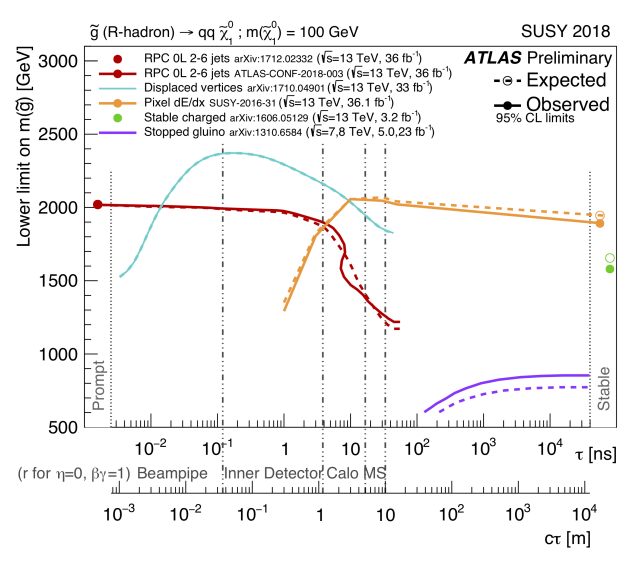

Figure 4: Constraints on the gluino mass-vs-lifetime plane for a splitsupersymmetry model with the gluino R-hadron decaying into a gluon or light quarks and a neutralino with a mass of $100 \mathrm{GeV}$. The solid lines indicate the observed limits, while the dashed lines indicate the expected limits. The area below the curves is excluded. For the displaced vertices result the expected and observed limits are identical. For the stopped gluino result the limit extends to larger lifetimes (not quoted here, see reference). The dots represent results for which the particle is assumed to be prompt or stable (escaping the detector) [3].

larly challenging and often the limiting factor. Three recent results exploit tracklets only in the Pixel detector looking for disappearing tracks [12], large-radius tracking algorithm to identify displaced vertices [13] and the correlation between b-tagging efficiency and lifetime to reinterpret more traditional SUSY searches [14], respectively. No evidence for new particles is found in any of the analyses and limits at $95 \%$ confidence level are set either on the mass or on the cross section as a function of the lifetime. The strongest limits are obtained for lifetimes of the order of 0.1 and $1 \mathrm{~ns}$, as shown in Figure 4. At the HL-LHC the combination of tracks and tracklets will lead to a full efficient reconstruction already for a chargino decay radius of $250 \mathrm{~mm}$, which together with the higher luminosity will extend the sensitivity to higher chargino masses for a given lifetime by a factor of about two [1].

\subsection{Signature-based and DM searches}

The most typical signature-based searches for BSM particles look for resonances in the form of a peak over a smooth background in the spectrum of a sensitive kinematic variable, e.g. the invariant mass of the decay products. Results compatible with the SM expectation are presented as (almost) model independent limits in crosssection times branching fraction $(\sigma \cdot B R)$, as well as model dependent limits in the mass of the new particle. At low invariant masses the limiting factor is the uncertainty on the background estimate, at high invariant masses the results are mostly statistically limited. Recent results include diboson searches for a resonance decaying to a $W, Z$ or Higgs boson $(h)$ together with a photon with a highly collimated (boosted) decay of the $W, Z$ or $h$ [15] and in the decay of a heavy neutral Higgs boson, $A$, decaying into a $Z$ boson and another heavy Higgs boson, $H$, in the context of the two-Higgsdoublet model (2HDM) [16]. Di-jet resonance searches were also recently published, for which either special trigger requirements are implemented to cover the lowmass region in the $b b$ or $b g$ decay channel [17], or the whole analysis is performed in the fast electronic of the first trigger level [18]. In none of the analyses an ex-

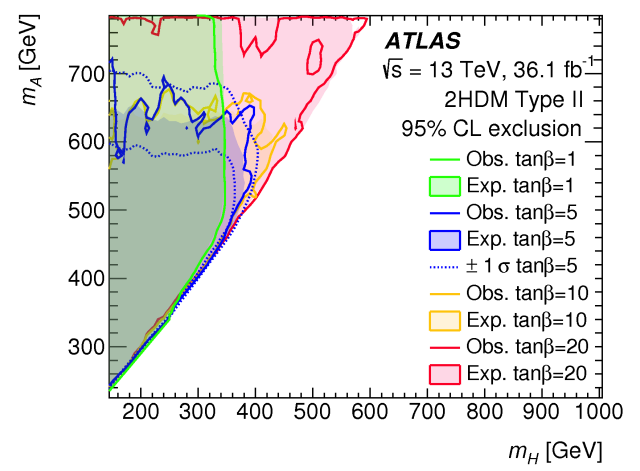

Figure 5: Observed and expected 95\% CL exclusion regions in the $\left(m_{A}, m_{H}\right)$ plane for various $\tan \beta$ values for Type II $2 \mathrm{HDM}$, obtained from the search for a heavy Higgs boson $(A)$ decaying into a $Z$ boson and another heavy Higgs boson $(H)[16]$.

cess with respect to the SM expectation is observed and 95\% confidence level limits on $\sigma \cdot B R$ as low as a fraction of a picobarn for resonance masses up to $6 \mathrm{TeV}$ are set and in some cases translated into model dependent mass limits. The results of the search for $A \rightarrow Z H$ in the $\left(m_{H}, m_{A}\right)$ plane for the 2HDM Type II model are shown in Figure 5. The expected sensitivity of a search for a $Z^{\prime}$ boson decaying to $t \bar{t}$ at the HL-LHC is presented in [19]. If no excess is found, the limit at $95 \%$ confidence level is expected to be at a $Z^{\prime}$ mass of $4 \mathrm{TeV}$.

Additionally to the RPC SUSY searches, that can be interpreted as DM searches, some dedicated analyses exploit a high $E_{T}^{\text {miss }}$ signature when looking for DM candidates, while resonance searches can be reinterpreted to set model dependent limits on mediator masses. A search for a single $b$ or $t$ quark with high $E_{T}^{\text {miss }}$ uses a very similar strategy to SUSY searches with lowbackground signal regions [20], while a search for a DM candidate accompanied by a vector boson has a large irreducible background and is limited by systematic uncertainties [21]. No evidence for an excess with 


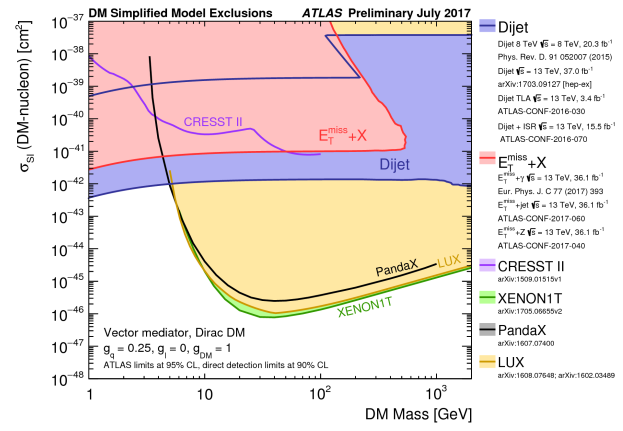

Figure 6: A comparison of the inferred limits to the constraints from direct detection experiments on the spin-independent WIMP-nucleon scattering cross section in the context of the $Z^{\prime}$-like simplified model with vector couplings. The results from this analysis, excluding the region to the left of the contour, are compared with limits from the LUX, PandaX, Xenon1T and CRESST II experiments. LHC limits are shown at $95 \%$ CL and direct detection limits at 90\% CL. The comparison is valid solely in the context of this model, assuming a mediator width fixed by the dark matter mass and coupling values $g_{q}=0.25$ and $g_{D M}=1$. LHC searches and direct detection experiments exclude the shaded areas. Exclusions of smaller scattering cross-sections do not imply that larger scattering cross-sections are also excluded. The single dijet and $E_{T}^{\text {miss }}+\mathrm{X}$ exclusion region represents the union of exclusions from all analyses of that type [3].

respect to the SM expectation is observed and $95 \%$ confidence limits are set in the DM particle vs. mediator mass plane. For a specific choice of model and couplings, these limits can be compared to the results from direct DM detection experiments, see Figure 6. While the direct detection experiments cover in particular very low cross sections at moderate DM candidate masses, the LHC sensitivity extends to much lower DM candidate masses for moderate cross sections. The expected sensitivity at the HL-LHC for the jet $+E_{T}^{\text {miss }}$ signature is investigated in [22], where a strong dependence is found on the systematic uncertainty of the SM background estimate.

\section{Indirect BSM searches}

An alternative approach to direct searches is to look for deviations from SM expectations either via enhancement of very rare processes (see Section 3.1) or via anomalous couplings (see Section 3.2).

\subsection{Very rare processes}

For some processes expected to occur with very low probability in the SM, a strong enhancement is predicted in BSM models. Therefore experimental searches are well motivated even long before the sensitivity to the SM cross section can be reached. These searches are statistically limited and they often make use of multivariate techniques to separate signal from background and of data-driven background estimates.

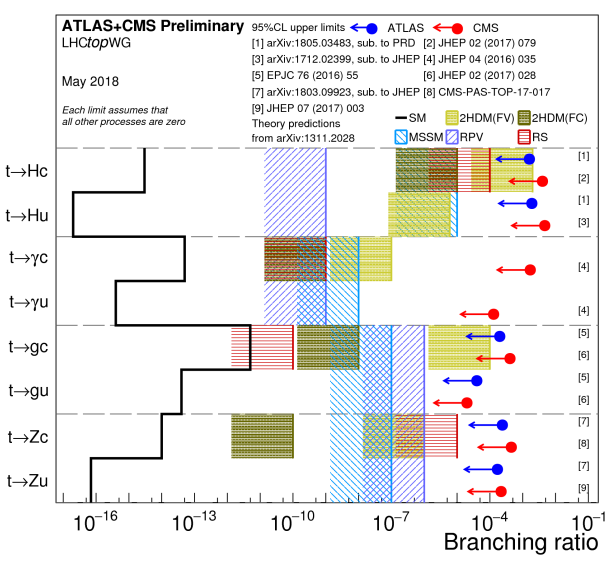

Figure 7: Summary of the current $95 \%$ confidence level observed limits on the branching ratios of the top quark decays via flavour changing neutral currents to a quark and a neutral boson $t \rightarrow X q(X=g, Z, \gamma$ or $H ; q=u$ or $c$ ) by the ATLAS and CMS Collaborations compared to several new physics models [3].

Flavour-changing neutral current (FCNC) decays of the top quark into a Higgs boson and a quark are excluded at $95 \%$ confidence level for branching fractions above $0.19 \%$ [23] with an expected improvement at the HL-LHC by more than an order of magnitude [24]. Figure 7 shows a summary of the limits on FCNC top quark decays set by the ATLAS and CMS experiments. The cross section of the four top quark production is excluded at $95 \%$ confidence level above 60 $\mathrm{fb}$, corresponding to 6.5 times the SM cross section [25]. Limits on the production cross section of two Higgs bosons decaying to a pair of $b$ quarks and a pair of photons are used to constrain the Higgs boson selfcoupling $\left(\kappa_{\lambda}=\lambda_{H H H} / \lambda_{H H H}^{\mathrm{SM}}\right)$ at $95 \%$ confidence level to $-8.2<\kappa_{\lambda}<13.2$ [26]. At the end of the HL-LHC running this limit is expected to improve to $-0.7<\kappa_{\lambda}<7.7$ [27] under the assumption that no significant deviation from the SM expectation is observed.

\subsection{Higgs boson and top quark couplings}

For a long time cross section measurements of several production and decay channels of the SM Higgs boson have been interpreted in terms of effective couplings within the so-called $\kappa$ framework, which is strongly model dependent $[30,31]$. Recently both inclusive and differential cross section measurements have been interpreted in terms of anomalous couplings within the context of effective field theories (EFT) [28]. As an exam- 
ple of the latter interpretation, the $68 \%$ and $95 \%$ confidence level regions for the Wilson coefficients specifying the strength of the new CP-even and CP-odd interactions to vector bosons from the measurement of the $H \rightarrow \gamma \gamma$ decay are shown in Figure 8. So far all cou-

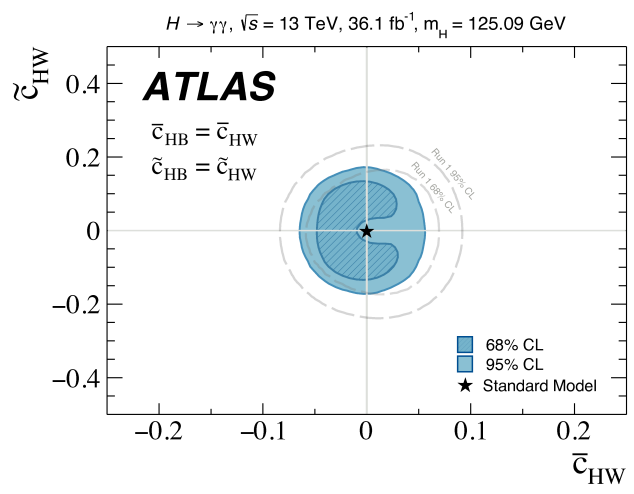

Figure 8: The observed 68\% (dark) and 95\% (light) confidence level regions from the simultaneous fit to the $\bar{c}_{\mathrm{HW}}$ and $\tilde{c}_{\mathrm{HW}}$ Wilson coefficients. The values of $\bar{c}_{\mathrm{HB}}$ and $\tilde{c}_{\mathrm{HB}}$ are set to be equal to $\bar{c}_{\mathrm{HW}}$ and $\tilde{c}_{\mathrm{HW}}$, respectively, and all other Wilson coefficients are set to zero. The SM expectation at $(0,0)$ is also shown, together with the Run 1 confidence regions [28].

plings have been found to be compatible with the SM prediction within uncertainty. The expected improvement in the coupling determination with the integrated luminosity accumulated at the end of Run 3 and at the end of the HL-LHC running is estimated within the $\kappa$ framework in [32]. Most ratios of couplings can reach the precision of a few to about $10 \%$.

Precise measurements of top quark properties, like the single top quark production in the $t$-channel [29] and the $W$ boson polarisation in top pair production [33] are used to constrain BSM contributions to the $W t b$ vertex within EFT. While in the SM at LO only the lefthanded vector coupling is non-vanishing, an effective Lagrangian can be built to include left- and right-handed vector $\left(V_{L}, V_{R}\right)$ and tensor $\left(g_{L}, g_{R}\right)$ couplings and limits are set on ratios of these complex coupling constants. Without any assumptions on the values of the other parameters, the most stringent constraints are obtained in [29] with the following 95\% confidence level intervals: $-0.12<\operatorname{Re}\left[g_{R} / V_{L}\right]<0.17$ and $-0.07<\operatorname{Im}\left[g_{R} / V_{L}\right]<$ 0.06 . These interpretations are currently based on results obtained from data at $\sqrt{s}=8 \mathrm{TeV}$.

\section{Conclusion}

An extensive programme of direct BSM searches is carried out at ATLAS employing a large variety of strategies and many ingenuous analysis methods. So far no deviations from the SM expectations have been observed and limits have been set on production cross section times branching fraction of several BSM particles as well as on their mass in the context of specific models. Additionally, more and more SM measurements are being interpreted as limits on anomalous couplings. Very rare processes are being searched for long before sensitivity at SM level is reached and Higgs boson and top quark cross section and property measurements are interpreted in the context of EFT.

With the high luminosity upgrade of the LHC a slightly higher energy, $3 \mathrm{ab}^{-1}$ of integrated luminosity and a strongly improved detector will be of great advantage for all statistically limited searches. At the same time the background estimate can profit from more populated control regions, as well as more precise measurements and calculations. On the other hand the much higher pile-up requires improved analysis techniques. Fortunately, already many very promising ideas have been developed in collaboration between experimentalists and theorists.

\section{References}

[1] ATLAS Collaboration, Technical Design Report for the ATLAS Inner Tracker Pixel Detector, Tech. Rep. CERN-LHCC-2017021. ATLAS-TDR-030, CERN, Geneva (Sep 2017). URL https : //cds . cern. ch/record/2285585

[2] ATLAS Collaboration, The ATLAS Experiment at the CERN Large Hadron Collider, JINST 3 (2008) S08003. doi:10.1088/1748-0221/3/08/S08003.

[3] ATLAS Collaboration, Directory of ATLAS Summary Plots. URL http: //cern.ch/go/mW9D

[4] ATLAS Collaboration, Search for supersymmetry in final states with charm jets and missing transverse momentum in $13 \mathrm{TeV}$ $p p$ collisions with the ATLAS detector, JHEP 09 (2018) 050. arXiv:1805.01649, doi:10.1007/JHEP09(2018)050.

[5] ATLAS Collaboration, Search for top squarks decaying to tau sleptons in $p p$ collisions at $\sqrt{s}=13 \mathrm{TeV}$ with the ATLAS detector, Phys. Rev. D98 (3) (2018) 032008. arXiv:1803.10178, doi:10.1103/PhysRevD.98.032008.

[6] ATLAS Collaboration, A search for pair-produced resonances in four-jet final states at $\sqrt{s}=13 \mathrm{TeV}$ with the ATLAS detector, Eur. Phys. J. C78 (3) (2018) 250. arXiv:1710.07171, doi:10.1140/epjc/s10052-018-5693-4.

[7] ATLAS Collaboration, Prospects for a search for direct pair production of top squarks in scenarios with compressed mass spectra at the high luminosity LHC with the ATLAS Detector, Tech. Rep. ATL-PHYS-PUB-2016-022, CERN, Geneva (Oct 2016). URL https : //cds . cern. ch/record/2220904

[8] ATLAS Collaboration, Prospect for a search for direct stau production in events with at least two hadronic taus and missing transverse momentum at theHigh Luminosity LHC with the ATLAS Detector, Tech. Rep. ATL-PHYS-PUB-2016-021, CERN, Geneva (Oct 2016).

URL http: //cds . cern. ch/record/2220805

[9] ATLAS Collaboration, Search for electroweak production of supersymmetric particles in final states with two or three leptons 
at $\sqrt{s}=13 \mathrm{TeV}$ with the ATLAS detector, Submitted to: EPJC. arXiv: 1803.02762 .

[10] ATLAS Collaboration, Search for supersymmetry in events with four or more leptons in $\sqrt{s}=13 \mathrm{TeV} p p$ collisions with ATLAS, Phys. Rev. D98 (3) (2018) 032009. arXiv:1804.03602, doi:10.1103/PhysRevD.98.032009.

[11] ATLAS Collaboration, Prospect for a search for direct pair production of a chargino and a neutralino decaying via a $\mathrm{W}$ boson and the lightest Higgs boson in final states with one lepton, two b-jets and missing transverse momentum at the high luminosity LHC with the ATLAS Detector., Tech. Rep. ATL-PHYS-PUB2015-032, CERN, Geneva (Jul 2015). URL http : //cds . cern. ch/record/2038565

[12] ATLAS Collaboration, Search for long-lived charginos based on a disappearing-track signature in pp collisions at $\sqrt{s}=$ $13 \mathrm{TeV}$ with the ATLAS detector, JHEP 06 (2018) 022. arXiv:1712.02118, doi:10.1007/JHEP06(2018)022.

[13] ATLAS Collaboration, Search for long-lived, massive particles in events with displaced vertices and missing transverse momentum in $\sqrt{s}=13 \mathrm{TeV} p p$ collisions with the ATLAS detector, Phys. Rev. D97 (5) (2018) 052012. arXiv:1710.04901, doi:10.1103/PhysRevD.97.052012.

[14] ATLAS Collaboration, Reinterpretation of searches for supersymmetry in models with variable $R$-parity-violating coupling strength and long-lived $R$-hadrons, Tech. Rep. ATLAS-CONF2018-003, CERN, Geneva (Mar 2018).

URL http : //cds . cern. ch/record/2308391

[15] ATLAS Collaboration, Search for heavy resonances decaying to a photon and a hadronically decaying $\mathrm{Z} / \mathrm{W} / \mathrm{H}$ boson in $p p$ collisions at $\sqrt{s}=13 \mathrm{TeV}$ with the ATLAS detector, Phys. Rev. D98 (3) (2018) 032015. arXiv:1805.01908, doi:10.1103/PhysRevD.98.032015.

[16] ATLAS Collaboration, Search for a heavy Higgs boson decaying into a $Z$ boson and another heavy Higgs boson in the $\ell \ell b b$ final state in $p p$ collisions at $\sqrt{s}=13 \mathrm{TeV}$ with the ATLAS detector, Phys. Lett. B783 (2018) 392-414. arXiv:1804.01126, doi:10.1016/j.physletb.2018.07.006.

[17] ATLAS Collaboration, Search for resonances in the mass distribution of jet pairs with one or two jets identified as $b$-jets in proton-proton collisions at $\sqrt{s}=13 \mathrm{TeV}$ with the ATLAS detector, Phys. Rev. D98 (2018) 032016. arXiv:1805.09299, doi:10.1103/PhysRevD.98.032016.

[18] ATLAS Collaboration, Search for low-mass dijet resonances using trigger-level jets with the ATLAS detector in $p p$ collisions at $\sqrt{s}=13 \mathrm{TeV}$, Phys. Rev. Lett. 121 (8) (2018) 081801. arXiv:1804.03496, doi:10.1103/PhysRevLett.121.081801.

[19] ATLAS Collaboration, Study on the prospects of a $t \bar{t}$ resonance search in events with one lepton at a High Luminosity LHC, Tech. Rep. ATL-PHYS-PUB-2017-002, CERN, Geneva (Feb 2017).

URL https : //cds . cern. ch/record/2243753

[20] ATLAS Collaboration, Search for dark matter produced in association with bottom or top quarks in $\sqrt{s}=13 \mathrm{TeV}$ pp collisions with the ATLAS detector, Eur. Phys. J. C78 (1) (2018) 18. arXiv:1710.11412, doi:10.1140/epjc/s10052-017-5486-1.

[21] ATLAS Collaboration, Search for dark matter in events with a hadronically decaying vector boson and missing transverse momentum in $p p$ collisions at $\sqrt{s}=13 \mathrm{TeV}$ with the ATLAS detector, Submitted to: JHEP. arXiv:1807.11471.

[22] ATLAS Collaboration, Sensitivity to WIMP Dark Matter in the Final States Containing Jets and Missing Transverse Momentum with the ATLAS Detector at $14 \mathrm{TeV}$ LHC, Tech. Rep. ATLPHYS-PUB-2014-007, CERN, Geneva (Jun 2014).

URL http: //cds . cern. ch/record/1708859

[23] ATLAS Collaboration, Search for flavor-changing neutral cur- rents in top quark decays $t \rightarrow H c$ and $t \rightarrow H u$ in multilepton final states in proton-proton collisions at $\sqrt{s}=13 \mathrm{TeV}$ with the ATLAS detector, Phys. Rev. D98 (3) (2018) 032002. arXiv:1805.03483, doi:10.1103/PhysRevD.98.032002.

[24] ATLAS Collaboration, Expected sensitivity of ATLAS to FCNC top quark decays $t \rightarrow \mathrm{Zu}$ and $t \rightarrow H q$ at the High Luminosity LHC, Tech. Rep. ATL-PHYS-PUB-2016-019, CERN, Geneva (Aug 2016).

URL http: //cds . cern. ch/record/2209126

[25] ATLAS Collaboration, Search for new phenomena in a lepton plus high jet multiplicity final state with the ATLAS experiment using $\sqrt{s}=13 \mathrm{TeV}$ proton-proton collision data, JHEP 09 (2017) 088. arXiv:1704.08493, doi:10.1007/JHEP09(2017)088.

[26] ATLAS Collaboration, Search for Higgs boson pair production in the $\gamma \gamma b \bar{b}$ final state with $13 \mathrm{TeV} p p$ collision data collected by the ATLAS experiment, Accepted by: JHEP. arXiv:1807.04873.

[27] ATLAS Collaboration, Study of the double Higgs production channel $H(\rightarrow b \bar{b}) H(\rightarrow \gamma \gamma)$ with the ATLAS experiment at the HL-LHC, Tech. Rep. ATL-PHYS-PUB-2017-001, CERN, Geneva (Jan 2017).

URL http: //cds . cern. ch/record/2243387

[28] ATLAS Collaboration, Measurements of Higgs boson properties in the diphoton decay channel with $36 \mathrm{fb}^{-1}$ of $p p$ collision data at $\sqrt{s}=13 \mathrm{TeV}$ with the ATLAS detector, Phys. Rev. D98 (2018) 052005. arXiv:1802.04146, doi:10.1103/PhysRevD.98.052005.

[29] ATLAS Collaboration, Analysis of the $W t b$ vertex from the measurement of triple-differential angular decay rates of single top quarks produced in the $t$-channel at $\sqrt{s}=8 \mathrm{TeV}$ with the ATLAS detector, JHEP 12 (2017) 017. arXiv:1707.05393, doi:10.1007/JHEP12(2017)017.

[30] ATLAS Collaboration, Measurement of the Higgs boson coupling properties in the $H \rightarrow Z Z^{*} \rightarrow 4 \ell$ decay channel at $\sqrt{s}=13 \mathrm{TeV}$ with the ATLAS detector, JHEP 03 (2018) 095. arXiv:1712.02304, doi:10.1007/JHEP03(2018)095.

[31] ATLAS Collaboration, Combined measurements of Higgs boson production and decay in the $H \rightarrow Z Z \rightarrow 4 \ell$ and $H \rightarrow \gamma \gamma$ channels using $\sqrt{s}=13 \mathrm{TeV}$ pp collision data collected with the ATLAS experiment, Tech. Rep. ATLAS-CONF-2017-047, CERN, Geneva (Jul 2017).

URL http: //cds . cern. ch/record/2273854

[32] ATLAS Collaboration, Projections for measurements of Higgs boson cross sections, branching ratios and coupling parameters with the ATLAS detector at a HL-LHC, Tech. Rep. ATL-PHYSPUB-2013-014, CERN, Geneva (Oct 2013). URL http://cds . cern. ch/record/1611186

[33] ATLAS Collaboration, Measurement of the W boson polarisation in $t \bar{t}$ events from pp collisions at $\sqrt{s}=8 \mathrm{TeV}$ in the lepton + jets channel with ATLAS, Eur. Phys. J. C77 (4) (2017) 264. arXiv:1612.02577, doi:10.1140/epjc/s10052-017-4819-4. 